\title{
Trends in Prevalence of Nonalcoholic Fatty Liver Disease in US Adults with Prediabetes
}

\author{
Phuc Le, PhD, $\mathrm{MPH}^{7}$, Alexander Chaitoff, MD, MPH${ }^{2}$, Michael B. Rothberg, MD, $M P H^{7}$, \\ Naim Alkhouri, $\mathrm{MD}^{3}$, and Arthur McCullough, $M D^{4,5}$

\begin{abstract}
${ }^{1}$ Center for Value-based Care Research, Medicine Institute, Cleveland Clinic, Cleveland, OH, USA; ${ }^{2}$ Department of Internal Medicine, Brigham and Women's Hospital, Boston, MA, USA; ${ }^{3}$ Texas Liver Institute, San Antonio, TX, USA; ${ }^{4}$ Digestive Disease and Surgery Institute, Cleveland Clinic, Cleveland, OH, USA; ${ }^{5}$ Department of Inflammation and Immunity, Lerner Institute, Cleveland Clinic, Cleveland, OH, USA.
\end{abstract}

J Gen Intern Med 34(11):2336-8

DOI: $10.1007 / \mathrm{s} 11606-019-05163-8$

(c) Society of General Internal Medicine 2019

\section{INTRODUCTION}

Prediabetes affects 84 million US adults, more than $50 \%$ of whom will develop diabetes in 15 years. ${ }^{1}$ Once patients develop diabetes, up to $75 \%$ will have nonalcoholic fatty liver disease (NAFLD), and many of these will develop nonalcoholic steatohepatitis (NASH) and advanced fibrosis. ${ }^{2}$ However, the burden of NAFLD and associated comorbidities in patients with prediabetes is less well understood. We aimed to estimate the population-based trends in prevalence of concurrent suspected NAFLD, advanced fibrosis and NASH cirrhosis in US adults with prediabetes.

\section{METHODS}

We conducted a cross-sectional, retrospective cohort analysis of the 2003-2014 National Health and Nutrition Examination Survey (NHANES). We included people aged $\geq 18$ years who had a $\mathrm{HbAlC}$ from 5.7 to $6.4 \%$ or fasting plasma glucose from 100 to $125 \mathrm{mg} / \mathrm{dL}$, or who were told they had prediabetes by a doctor but had never been diagnosed with diabetes. Patients with other causes of liver disease: viral hepatitis, excess alcohol consumption ( $>1$ drink/day for women, $>2$ drinks/day for men); aspartate aminotransferase (AST) or alanine aminotransferase (ALT) > 500; pregnancy; or missing data were excluded. Suspected NAFLD was defined alternatively as hepatic steatosis index (HSI) $>36$, fatty liver index (FLI) $\geq$ 60 , either, or both. ${ }^{3}$ NAFLD-associated advanced fibrosis was defined as BMI $\geq 25 \mathrm{~kg} / \mathrm{m}^{2}$, ALT $>40 \mathrm{U} / \mathrm{L}$ (men), or $>30 \mathrm{U} / \mathrm{L}$ (women) and either AST-to-platelet ratio index (APRI) $>1$, fibrosis-4 (FIB-4) index $>2.67$, or NAFLD fibrosis score (NFS) $>0.676 .{ }^{4}$ Cirrhosis was defined as APRI $>2$ and either ALT $>40 \mathrm{U} / \mathrm{L}$ (men) or $>30 \mathrm{U} / \mathrm{L}$ (women), alkaline phosphatase $>113 \mathrm{U} / \mathrm{L}$, or total bilirubin $>1.3 \mathrm{mg} / \mathrm{dL}$, and NASH cirrhosis as cirrhosis plus either obesity, insulin resistance, or metabolic syndrome. ${ }^{4}$

To improve precision, the prevalence of each condition was estimated in 4-year periods: 2003-2006, 2007-2010, and

Published online July 19, 2019
2011-2014. We used logistic regression to examine trends, accounting for the complex survey design of NHANES.

\section{RESULTS}

Our final sample included 19,902 adults, of whom 7209 (36.2\%) had prediabetes but only 4094 were included in the estimation of NASH cirrhosis due to missing data. Prevalence of prediabetes rose from 30.5 to $34.8 \%$ (p value $=0.009)$ while prevalence of concurrent prediabetes and suspected NAFLD remained stable ( $p$ value $>0.05$ ) (Table 1). In prediabetic patients, prevalence of suspected NAFLD decreased significantly from 2003 to 2014 if suspected NAFLD was defined by FLI and by either FLI or HSI, but not by HSI and by both scores. Prevalence of NAFLD-associated advanced fibrosis did not change over time. Overall prevalence of NASH cirrhosis was $0.12 \% 95 \%$ CI: (-0.05)-0.28\%. Translating to the general population, between 27 and 38 million US adults with prediabetes had suspected NAFLD (Fig. 1) and 420,000 patients had advanced fibrosis..

\section{DISCUSSION}

In this population-based study, we found that between 44 and $62 \%$ of US adults with prediabetes had concurrent suspected NAFLD, equivalent to 27-38 million patients, and similar to the numbers of prediabetic patients with hypertension or dyslipidemia. Our study appears to be the first to establish a populationbased estimate of suspected NAFLD prevalence trends in prediabetic US adults. In Germany, the prevalence of NAFLD measured by localized proton magnetic resonance spectroscopy was $45-78 \%,{ }^{5}$ while in Korea, it was $60 \%$ based on ultrasound. ${ }^{6}$

The prevalence of prediabetes in the USA has been increasing for two decades, reaching $34 \%$ in 2015 . Although intensive lifestyle modification and metformin have been shown to reduce progression to diabetes by $27 \%$ and $18 \%$, respectively, ${ }^{1}$ it is not known whether these interventions can affect liver function and prevent NAFLD progression. It is also not known to what extent these interventions are being used in clinical practice. Our findings that at least 27 million US adults have 
Table 1 Prevalence (95\% CI) of Suspected NAFLD and Advanced Fibrosis by Different Definitions Among US Adults, 2003-2014

\begin{tabular}{|c|c|c|c|c|}
\hline & $2003-2006$ & $2007-2010$ & 2011-2014 & $p$ value for trends \\
\hline Total sample size & 5783 & 7264 & 6855 & \\
\hline Prevalence of prediabetes $(\%)$ & $30.5[28.1-33.0]$ & $37.2[36.0-38.3]$ & $34.8[32.8-36.8]$ & 0.009 \\
\hline \multicolumn{5}{|c|}{ Prevalence of concurrent prediabetes and suspected NAFLD in the general population $(\%) *$} \\
\hline HSI $>36$ & $15.9[14.0-17.7]$ & $16.7[15.4-18.0]$ & $15.1[13.8-16.5]$ & 0.498 \\
\hline FLI $\geq 60$ & $13.8[12.1-15.5]$ & $13.4[12.3-14.4]$ & $12.1[10.7-13.5]$ & 0.138 \\
\hline $\mathrm{HSI}>36$ or $\mathrm{FLI} \geq 60$ & $17.1[15.3-19.0]$ & $17.8[16.6-19.1]$ & $15.9[14.3-17.4]$ & 0.335 \\
\hline $\mathrm{HSI}>36$ and $\mathrm{FLI} \geq 60$ & $12.6[10.9-14.3]$ & $12.2[11.1-13.3]$ & $11.3[10.1-12.5]$ & 0.226 \\
\hline \multicolumn{5}{|c|}{ Prevalence of suspected NAFLD in the prediabetic population $(\%)^{\dagger}$} \\
\hline HSI $>36$ & $61.0[57.7-64.4]$ & $56.5[53.5-59.5]$ & $56.9[53.2-60.6]$ & 0.112 \\
\hline FLI $\geq 60$ & $53.2[49.1-57.2]$ & $45.4[42.5-48.2]$ & $45.7[41.7-49.7]$ & 0.013 \\
\hline HSI $>36$ or FLI $\geq 60$ & $65.5[62.7-68.3]$ & $60.4[57.6-63.2]$ & $59.8[56.2-63.3]$ & 0.015 \\
\hline $\mathrm{HSI}>36$ and FLI $>60$ & $48.7[44.2-53.1]$ & $41.5[38.5-44.5]$ & $42.8[38.9-46.7]$ & 0.059 \\
\hline \multicolumn{5}{|c|}{ Prevalence of NAFLD-associated advanced fibrosis in the prediabetic population $(\%)^{\dagger}$} \\
\hline Any score & $0.67[0.18-1.17]$ & $0.54[0.19-0.89]$ & $0.77[0.21-1.33]$ & 0.779 \\
\hline APRI & $0.38[-0.02-0.77]$ & $0.36[0.08-0.64]$ & $0.29[-0.17-0.76]$ & 0.79 \\
\hline FIB & $0.27[-0.04-0.57]$ & $0.18[0.01-0.36]$ & $0.38[-0.1-0.85]$ & 0.678 \\
\hline NFS & $0.18[-0.01-0.37]$ & $0.41[0.11-0.71]$ & $0.48[0.12-0.83]$ & 0.139 \\
\hline
\end{tabular}

*The prevalence was estimated among the general population

tThe prevalence was estimated among the subset of prediabetic patients without any other causes of fatty liver. APRI, aspartate aminotransferasel platelet ratio index; CI, confidence interval; FLI, fatty liver index; HSI, hepatic steatosis index; NFS, nonalcoholic fatty liver disease fibrosis score; FIB4, fibrosis-4 index

prediabetes and NAFLD suggests a coming epidemic of advanced liver complications if urgent prevention measures are not undertaken.

Our study is limited by the lack of biopsy and imaging data to confirm NAFLD-associated diagnosis. Nonetheless, we used two different noninvasive scores and different ways to define suspected NAFLD so as to provide conservative estimates, which were consistent with published studies. Addi- tional limitations are the exclusion of normal BMI or liver enzyme in the definition of advanced fibrosis and limited sample size for the NASH cirrhosis estimate.

In conclusion, at least half of US adults with prediabetes had suspected NAFLD - a prevalence similar to that in diabetes. Pharmacological and nonpharmacological interventions that can address both diabetes development and NAFLD progression would be beneficial to the prediabetic population.

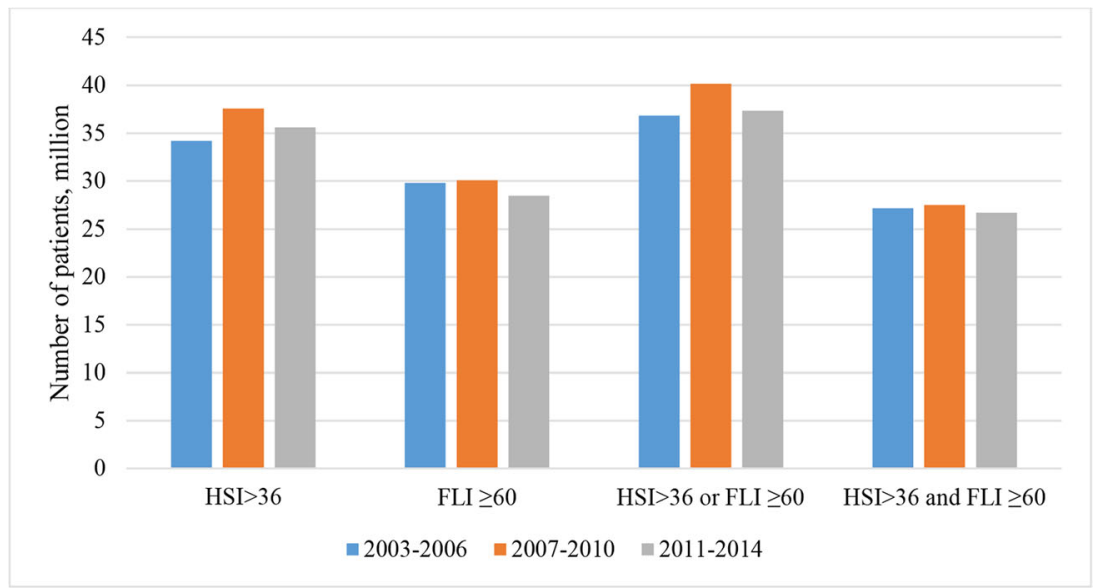

Figure 1 Population estimates of US adults with concurrent prediabetes and suspected NAFLD by different definitions of NAFLD, 2003-2014. FLI, fatty liver index; HSI, hepatic steatosis index; NAFLD, nonalcoholic fatty liver diseases.> 
Corresponding Author: Phuc Le, PhD, MPH; Center for Value-based Care Research, Medicine Institute Cleveland Clinic, Cleveland, $\mathrm{OH}$, USA (e-mail: lep@ccf.org).

\section{Compliance with Ethical Standards:}

Conflict of Interest: Dr. Alkhouri received research funding from Gilead, Intercept, Allergan, Cirius, Madrigal, and Genfit which was not related to this study. Dr. McCullough was supported by grant UO1 DK061732. All other authors have no conflicts of interest.

\section{REFERENCES}

1. Diabetes Prevention Program Research Group. Long-term effects of lifestyle intervention or metformin on diabetes development and microvascular complications over 15-year follow-up: the Diabetes Prevention Program Outcomes Study. Lancet Diabetes Endocrinol 2015;3(11):866-875.

2. Williams CD, Stengel J, Asike MI, et al. Prevalence of nonalcoholic fatty liver disease and nonalcoholic steatohepatitis among a largely middle-aged population utilizing ultrasound and liver biopsy: a prospective study. Gastroenterology. 2011;140(1):124-131.

3. Sviklane L, Olmane E, Dzerve Z, Kupcs K, Pirags V, Sokolovska J. Fatty liver index and hepatic steatosis index for prediction of non-alcoholic fatty liver disease in type 1 diabetes. J Gastroenterol Hepatol 2018;33(1):270276.

4. Kabbany MN, Conjeevaram Selvakumar PK, Watt K, et al. Prevalence of Nonalcoholic Steatohepatitis-Associated Cirrhosis in the United States: An Analysis of National Health and Nutrition Examination Survey Data. Am J Gastroenterol 2017;112(4):581-587.

5. Stefan N, Fritsche A, Schick F, Haring HU. Phenotypes of prediabetes and stratification of cardiometabolic risk. Lancet Diabetes Endocrinol 2016:4(9):789-798.

6. Bae JC, Cho YK, Lee WY, et al. Impact of nonalcoholic fatty liver disease on insulin resistance in relation to HbAlc levels in nondiabetic subjects. Am J Gastroenterol 2010;105(11):2389-2395.

Publisher's Note Springer Nature remains neutral with regard to jurisdictional claims in published maps and institutional affiliations. 